\title{
Nível de conhecimento de idosos comunitários em relação ao HIV/Aids: estudo exploratório na rede básica de saúde de Belém, Pará, Brasil
}

\author{
Rodolfo Gomes do Nascimento", Evanildo Lopes Monteiro", Laiana Soeiro Ferreira"*;, \\ Zeneide Nazaré Lima dos Santos ${ }^{* * *}$
}

\section{Resumo}

A população mundial está envelhecendo gradativamente, e em vista disso tem se notado inúmeras mudanças comportamentais na sexualidade dos idosos. Diante de tal panorama, faz-se necessário compreender melhor o processo de envelhecimento e suas relações com uma vida sexual ativa nessa faixa etária. Para tanto, o presente estudo objetivou avaliar o nível de conhecimento de idosos em relação ao HIV/à Aids. Trata-se de um estudo descritivo populacional com método quantitativo-exploratório que envolveu 310 idosos de ambos os sexos, atendidos em serviços de saúde da rede pública do município de Belém, Pará, Brasil. Para a coleta dos dados, utilizou-se um questionário semiestruturado sobre HIV/Aids, incluindo características gerais e informações sobre conceito, transmissão, prevenção, vulnerabilidade e tratamento do HIV/Aids. Os resultados encontrados e discutidos nesta pesquisa revelaram, em suma, o baixo nível de conhecimento, principalmente em relação aos questionamentos sobre conceitos, formas de transmissão e vulnerabilidade. Tais lacunas demonstram a necessidade de se implementar programas educativos específicos voltados para esse grupo etário na rede de saúde pública.

Palavras-chave: HIV. Conhecimento. Saúde do idoso. Epidemiologia

\section{Introdução}

Uma das mais importantes mudanças demográficas que o Brasil experimentou, ao encerrar o século XX, foi o acentuado envelhecimento da estrutura

* Mestre em Doenças Tropicais, Núcleo de Medicina Tropical, pela Universidade Federal do Pará (UFPA), Belém - PA. Especialista em Gerontologia pela FHCGV/UFPA. Fisioterapeuta da Secretaria Municipal de Saúde de Belém e Secretaria de Estado de Saúde Pública do Pará.

** Doutorando em Educação pela Universidade Federal do Pará. Docente da Faculdade de Fisioterapia e Terapia Ocupacional da mesma instituição. Endereço para correspondência: Rua Liberato de Castro, 532, Guamá, CEP: 66045-420, Belém - PA, Brasil. E-mail: rodgn@hotmail.com.

**** Mestranda em Psicologia pelo Programa de Teoria e Pesquisa do Comportamento. Docente da Faculdade de Terapia Ocupacional da Universidade Estadual do Pará e da Universidade da Amazônia.

***** Terapeuta Ocupacional do Centro de Atenção Psicossocial e do Hospital Municipal de Ulianópolis, Pará.

$\rightarrow$ http://dx.doi.org/10.5335/rbceh.2013.3018 
etária da população, o que trouxe como consequência uma maior presença relativa e absoluta da população de 60 anos e mais no país. (MIYATA et al., 2005; SOUSA, 2008).

Segundo Sousa (2008), muitos investimentos têm sido feitos para melhorar a qualidade de vida dos idosos, que começam a desfrutá-la em sua plenitude, devido à ampliação das relações sociais e, por que não, sexuais, já que a libido não acaba com o aumento da idade. Com isso, riscos antes característicos de faixas etárias mais jovens estão acometendo cada vez mais indivíduos com idade acima dos 60 anos.

$\mathrm{Na}$ atualidade, têm-se percebido mudanças no curso das infecções sexualmente transmissíveis (ISTs) e da epidemia de Aids (Acquired Immunodeficiency Syndrome), dentre as quais, o aumento do número de casos entre idosos. (SOUSA, 2008; OLIVI; SANTANA; MATHIAS, 2008). A Aids surgiu no início da década de 1980, tendo o primeiro caso no Brasil sido diagnosticado na cidade de São Paulo. As primeiras ocorrências dessa doença, que logo se tornou uma epidemia mundial, deram-se entre homossexuais e indivíduos submetidos a transfusões de sangue, seguidos pelo aparecimento de casos em usuários de drogas injetáveis, porém, hoje a Aids atinge a população em geral. A população idosa, de início, praticamente não foi atingida pela Aids. À época, considerava-se que os idosos tinham uma vida sexual inativa. (GODOY et al., 2008).

Pottes et al. (2007) afirmam que a problemática do envelhecimento e da Aids no Brasil passa por uma questão cultural e de exclusão e concentra-se, principalmente, no preconceito social relacionado ao sexo nessa idade. A prevenção às infecções sexualmente transmissíveis e à Aids entre os maiores de 50 anos é algo muito complexo e representa um desafio para as atuais políticas de saúde pública, que concentram sua atenção na população jovem (entre 20 e 34 anos).

Nesse contexto, com a estimativa do aumento expressivo no número de idosos, surge um grande desafio para o Brasil: o estabelecimento de políticas públicas e estratégias que possam garantir a qualidade de vida dessas pessoas. Portanto, questões como a Aids e o envelhecimento devem ser aprofundadas de modo a fornecer subsídios tanto para os cuidados com os portadores do HIV e da Aids, quanto para o desenvolvimento de ações e programas de prevenção. (POTTES et al., 2007).

De acordo com Lazzarotto et al. (2008), a literatura enfatiza o conhecimento sobre HIV/Aids em indivíduos jovens e profissionais da saúde; porém, há uma falta de informações relacionadas à Aids em idosos. Diante disso, torna-se extremamente importante identificar o nível de conhecimento dos idosos em relação ao HIV/à Aids, pois o conhecimento é importante não apenas para medidas de prevenção como também para a diminuição do preconceito com portadores do HIV.

Sendo assim, essa investigação propôs-se a avaliar o conhecimento sobre HIV/Aids nos idosos atendidos em um dos maiores programas de atenção à saúde do idoso da rede pública do município de Belém, Pará, Brasil. 


\section{Material e métodos}

Caracterizou-se o estudo como descritivo populacional com método quantitativo-exploratório. A amostragem por conveniência desta pesquisa foi composta por idosos, considerando a definição do Ministério da Saúde, que especifica como população idosa aquela a partir de 60 anos de idade. (BRASIL, 2003). Os critérios de inclusão estabelecidos foram: pacientes atendidos no Programa de Atenção à Saúde do Idoso Marambaia, regularmente matriculados, de ambos os sexos e que aceitaram cooperar com a realização da pesquisa por meio da autorização formalizada no Termo de Consentimento Livre e Esclarecido (TCLE). Foram excluídos da amostra todos os questionários que apresentaram falhas de registro (ausência ou não legibilidade de pelo menos uma informação).

Os dados foram coletados pelos pesquisadores entre junho e setembro de 2010 mediante um questionário semiestruturado sobre HIV/Aids para terceira idade (QHIV3I/adapt.). Lazzarotto et al. (2008) elaboraram esse questionário, avaliando sua fidedignidade por meio do coeficiente Kappa, e evidenciaram uma boa confiabilidade para idosos brasileiros de ambos os sexos. Considerando a regionalidade linguística, foi necessário modificar alguns termos do questionário para aplicação na região do presente estudo.

$\mathrm{O}$ instrumento utilizado abrange características gerais dos participantes e questões relativas ao HIV/à Aids, organizadas nos domínios "conceito", "transmissão", "prevenção", "vulnerabilidade" e "tratamento". As opções de resposta disponíveis foram: verdadeiro, falso e não sei. Entre as últimas perguntas do questionário, algumas incluíam a Aids como um castigo divino, o conhecimento de alguma pessoa infectada pelo HIV, a utilização de preservativo e a realização de teste anti-HIV. O preenchimento do QHIV3I/adapt. foi realizado em reuniões agendadas previamente entre $o$ pesquisador e os grupos (máximo 10 idosos), que foram obtidos via amostragem consecutiva.

Após a coleta dos dados, foi construído um banco de dados no programa $\mathrm{Mi}$ crosoft Office Excel 2007 do Windows ${ }^{\circledR}$, e para efetivar a análise das variáveis por meio da estatística descritiva, foi utilizado o programa Epi-Info ${ }^{\circledR}$ 6.04, de onde foram concluídas as distribuições de frequência e as tabelas.

Esta pesquisa foi desenvolvida somente após a apreciação e aprovação pela Comissão de Análise de Projeto de Pesquisa da SESMA/CAPP e pelo Comitê de Ética em Pesquisa da Universidade Federal do Pará (Parecer no 049/2010), estando de acordo com a Resolução $\mathrm{n}^{\mathrm{o}}$ 196/96 do Conselho Nacional de Saúde.

\section{Resultados}

A Tabela 1 apresenta as variáveis envolvendo as características gerais dos 310 idosos que compuseram a amostra da pesquisa. Destes, 82 eram homens $(26,4 \%)$ e 228 eram mulheres $(73,5 \%)$, com idade média de 74 anos e desvio padrão de 7,54, variando entre 60 e 83 anos. Quanto à escolaridade, $45,4 \%$ relataram ter cursado de quatro a sete anos de estudo; a renda mensal de $48,7 \%$ foi de um a três salários-mínimos; $65,1 \%$ referiram a prática da religião católica e 57,4\% não possuíam companheiro(a). 
Tabela 1. Características gerais dos idosos participantes da pesquisa $(n=310)$

\begin{tabular}{lrr}
\hline \multicolumn{1}{r}{ Variáveis } & $\mathrm{N}$ & $\%$ \\
\hline Sexo & & \\
Masculino & 82 & 26,4 \\
Feminino & 228 & 73,5
\end{tabular}

Escolaridade

Nenhuma

1 a 3 anos

114

4 a 7 anos

8 ou mais anos

Renda mensal

Até 1 salário-mínimo

1 a 3 salários-mínimos

151

4 a 6 salários-mínimos

Mais de 7 salários-mínimos

Religião

Católica

202

65,1

Evangélica

86

Outras

Nenhuma

Companheiro (a)

Sim

Não
Na Tabela 2, são apresentados os resultados relacionados aos conhecimentos dos idosos. Os resultados obtidos na pesquisa revelaram o baixo nível de conhecimento em relação aos conceitos, às formas de prevenção, de transmissão e vulnerabilidade em relação ao HIV/Aids em idosos usuários do serviço público de saúde do município de Belém.

Pôde-se constatar com a pesquisa, nos domínios "conceito" e "transmissão", que 57,7\% desconheciam a fase assintomática da infecção pelo HIV e que $47,4 \%$ acreditavam que a Aids poderia ser transmitida pelo "carapanã" (Tabela 2).

Em relação aos domínios "prevenção" e "vulnerabilidade", 18,4\% não acreditam que o uso da camisinha nas relações sexuais impede a transmissão do HIV, $26,4 \%$ não sabiam da existência da ca41,3 misinha feminina e $39 \%$ consideravam a Aids uma síndrome somente de homens que fazem sexo com homens, profissionais do sexo e usuários de drogas. Quanto ao "tratamento", 18,4\% ignoravam a sua existência (Tabela 2).

Com a pesquisa, observou-se que, entre os idosos, 23,8\% julgavam a Aids como um castigo divino para aqueles que cometeram pecados. Do total de idosos pesquisados, 36,7\% (114) conheciam alguma pessoa infectada pelo HIV, 88,4\% (274) não usavam preservativo e apenas 8,4\% (26) já tinham realizado o teste anti-HIV. 
Tabela 2. Conhecimentos dos idosos participantes do estudo $(n=310)$

\begin{tabular}{|c|c|c|c|c|c|c|}
\hline \multirow{2}{*}{ Variáveis } & \multicolumn{2}{|c|}{ Verdadeiro } & \multicolumn{2}{|c|}{ Falso } & \multicolumn{2}{|c|}{ Não sei } \\
\hline & $\mathrm{n}$ & $\%$ & $\mathrm{n}$ & $\%$ & $\mathrm{n}$ & $\%$ \\
\hline \multicolumn{7}{|l|}{ Domínio "Conceito" } \\
\hline O vírus HIV é o causador da Aids? & 228 & 73,5 & 39 & 12,6 & 43 & 13,9 \\
\hline $\begin{array}{l}\text { A pessoa com o vírus da Aids sempre apresen- } \\
\text { ta os sintomas da doença? }\end{array}$ & 179 & 57,7 & 58 & 18,7 & 73 & 23,5 \\
\hline $\begin{array}{l}\text { O vírus da Aids é identificado através de exa- } \\
\text { mes de laboratório? }\end{array}$ & 243 & 78,4 & 24 & 7,7 & 43 & 13,9 \\
\hline \multicolumn{7}{|l|}{ Domínio “Transmissão” } \\
\hline $\begin{array}{l}\text { O vírus da Aids pode ser transmitido pelo uso } \\
\text { de sabonetes, toalhas e sanitários? }\end{array}$ & 114 & 36,7 & 185 & 59,7 & 11 & 3,5 \\
\hline $\begin{array}{l}\text { O vírus da Aids pode ser transmitido por abra- } \\
\text { ço, beijo no rosto ou beber no mesmo copo? }\end{array}$ & 73 & 23,5 & 202 & 65,1 & 35 & 11,3 \\
\hline $\begin{array}{l}\text { O vírus da Aids pode ser transmitido por picada } \\
\text { de "carapanã"? }\end{array}$ & 147 & 47,4 & 112 & 36,1 & 51 & 16,4 \\
\hline \multicolumn{7}{|l|}{ Domínio “Prevenção" } \\
\hline $\begin{array}{l}\text { A pessoa que usa camisinha nas relações se- } \\
\text { xuais impede a transmissão do vírus da Aids? }\end{array}$ & 258 & 83,2 & 37 & 12 & 15 & 4,8 \\
\hline $\begin{array}{l}\text { Existe uma camisinha específica para as mu- } \\
\text { lheres? }\end{array}$ & 203 & 65,5 & 82 & 26,4 & 25 & 8 \\
\hline $\begin{array}{l}\text { O uso da mesma seringa e agulha por diversas } \\
\text { pessoas transmite Aids? }\end{array}$ & 264 & 85,1 & 35 & 11,3 & 11 & 3,5 \\
\hline \multicolumn{7}{|l|}{ Domínio "Vulnerabilidade" } \\
\hline $\begin{array}{l}\text { A Aids é uma doença que ocorre somente em } \\
\text { homens gays, prostitutas(os) e drogados? }\end{array}$ & 121 & 39 & 170 & 54,8 & 19 & 6,1 \\
\hline $\begin{array}{l}\text { Os idosos não devem se preocupar com a } \\
\text { Aids, pois ela atinge apenas os jovens? }\end{array}$ & 86 & 27,7 & 209 & 67,4 & 15 & 4,8 \\
\hline \multicolumn{7}{|l|}{ Domínio “Tratamento” } \\
\hline A Aids é uma doença que tem tratamento? & 218 & 70,3 & 57 & 18,4 & 35 & 11,3 \\
\hline A Aids é uma doença que tem cura? & 43 & 13,9 & 223 & 72 & 44 & 14,2 \\
\hline
\end{tabular}


Figura 1. Principais perguntas e percentuais de erro

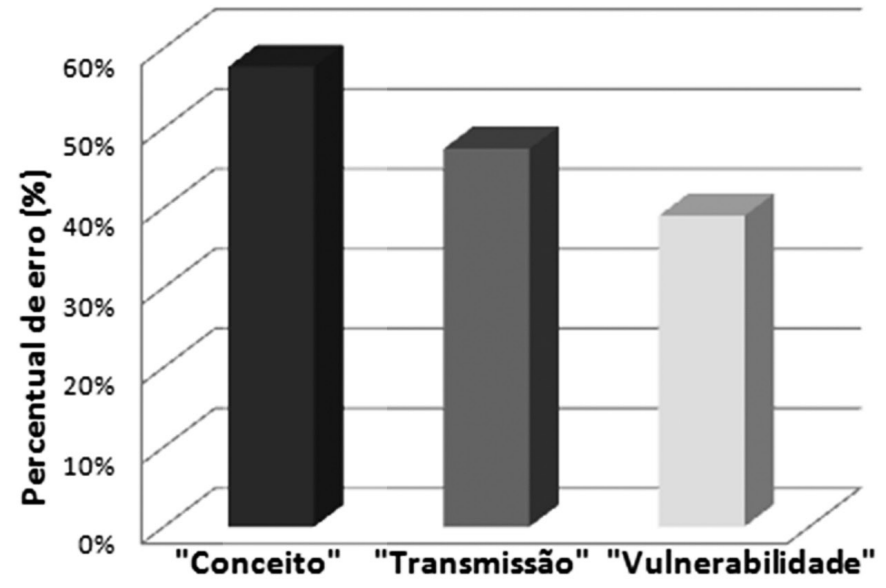

Perguntas

A pessoa com o vírus da aids sempre apresenta os sintomas da doença?

O vírus da aids pode ser transmitido por picada de "carapanâ"?

A aids é uma doença que ocorre somente em homens gays, prostitutas (os) e drogados?
Na Figura 1, destacam-se os resultados referentes às perguntas com maiores percentuais de erro, as quais compõem os domínios "Conceito", "Transmissão" e "Vulnerabilidade".

\section{Discussão}

Sabe-se que avaliar o conhecimento sobre o HIV/Aids é relevante, uma vez que este é considerado um determinante para risco comportamental. Afinal, o conhecimento incorporado pelo ser humano está associado à sua percepção de vulnerabilidade a um risco. (VERAS; CAMARGO, 1995).

Observa-se que a caracterização epidemiológica apresentada na pesquisa segue fidedignamente o perfil de idosos usuários da rede pública de saúde, em sua maioria mulheres, de baixa escolaridade e baixa renda. A tendência da epidemia do HIV/Aids entre os idosos segue essa mesma configuração, segundo algumas pesquisas já realizadas no Bra- sil. (POTTES et al., 2007; SALDANHA; FELIX; ARAÚJO, 2008).

Percebe-se que, em relação ao "conceito" de HIV e Aids, ainda hoje, uma grande parcela da população desconhece a diferença entre a infecção pelo vírus (HIV) e a manifestação da doença (Aids). Essa lacuna é preocupante, pois, quando o indivíduo infectado manifesta algum sintoma, seu sistema imunológico já está debilitado, com contagens de T CD4+ abaixo de 500 células/ $/ \mathrm{L}$. (ABBAS; LICHTMAN; PILLAI, 2000).

Um estudo realizado por Ferro e Salit (1992) com pacientes idosos portadores de HIV mostrou que a Aids nessa população específica manifesta-se por sinais e sintomas que, frequentemente, são confundidos com os de outras patologias, contribuindo, assim, para a demora no diagnóstico, que muitas vezes é realizado somente após uma longa investigação e/ ou por exclusão de outras doenças. De acordo com Santos et al. (2002), outros 
autores apontam, ainda, que, por preconceito, os próprios médicos não solicitam a sorologia para o HIV prontamente e que os idosos consideram-se imunes ao vírus. Além disso, os profissionais da área de saúde nem mesmo indagam os idosos sobre a vida sexual.

Com base nos dados do domínio "transmissão", observou-se que ainda há dúvida em relação às formas de transmissão do HIV, pois $47,4 \%$ dos indivíduos acreditavam que esta poderia ocorrer pela picada de "carapanã". O mesmo foi observado no estudo realizado em 2008 com idosos do Rio Grande do Sul, que indicaram uma frequência de $41,1 \%$, e nos estudos realizados com os moradores de uma favela do Rio de Janeiro em $1992(45,2 \%)$ e em 1998 (41,1\%). (LAZZAROTTO et al., 2008; FERNANDES; COUTINHO; MATIDA, 1992; FERNANDES, 1998).

De acordo com Bertoncini, Moraes e Kulkamp (2007), a transmissão do vírus HIV pode ocorrer pelas vias sexual, sanguínea, parenteral, ocupacional e vertical (materno-infantil). Sabe-se que o mosquito não pode ser considerado vetor na transmissão do HIV, tendo em vista que apresenta baixa infectividade, curta sobrevivência do vírus no mosquito e ausência do antígeno T4 na superfície celular dos artrópodes. (IQBAL, 1999).

No domínio "prevenção", 83,2\% dos idosos respondeu que a transmissão do HIV pode ser impedida com o uso de preservativos; no entanto, 88,4\% não faziam uso nas relações sexuais. Vários supostos motivos podem justificar tal negligência nessa faixa etária. Entre as mulheres, destaca-se o fato de estarem no período pós-menopausa, ou seja, já perderam a capacidade de engravidar e consideram que não necessitam mais de prevenção, não insistindo no uso do preservativo com seu parceiro. Vale ressaltar, também, que o sexo sem camisinha é particularmente arriscado depois da menopausa, devido ao ressecamento das paredes vaginais, quando as queixas tornam-se mais frequentes, podendo favorecer o surgimento de lesões que facilitam a infecção pelo vírus HIV. Já os homens temem perder a ereção e ainda acham que o cuidado somente é necessário nas relações com as profissionais do sexo. (LÔBO, 2011; POTTES et al., 2007; LEITE; MOURA; BERLEZ, 2007; SANTOS; ASSIS, 2011).

Segundo Lôbo (2011) e Sousa (2008), uma vez que a população idosa iniciou a vida sexual antes do surgimento do HIV/ Aids, não reconhece o risco de contrair a infecção, nem está familiarizada com o uso do preservativo. Nesse contexto, também se destaca o fator "fidelidade", que, não raro, é "provada" no tocante ao uso do preservativo, o qual historicamente esteve atrelado às dSTs e à desconfiança em traição psicoafetiva. Tal fato dificulta o diálogo acerca da necessidade do uso do preservativo, como se este somente devesse ser usado por pessoas que não se conhecem e/ou não têm uma relação de confiança mútua. (LEITE; MOURA; BERLEZ, 2007).

O Ministério da Saúde esclarece que a principal forma de prevenção da infecção pelo HIV é a utilização do preservativo, tanto masculino como o feminino, os quais são distribuídos gratuitamente em todas as unidades básicas de saúde e nos 
centros de testagem e aconselhamento de cada município. (BRASIL, 2005).

No que se refere à "vulnerabilidade", $39 \%$ consideravam a Aids uma síndrome somente de homens que fazem sexo com homens, profissionais do sexo e usuários de drogas. Essa noção de doença contraída somente por grupos específicos reflete muito bem a pandemia do HIV no início da década de 1980, quando esta foi descoberta, causando um grande impacto social, estigmas e preconceitos na época. No entanto, a epidemia de HIV/ Aids vem sofrendo diversas modificações em seu perfil ao longo do tempo, dentre as quais, os fenômenos de feminilização, heterossexualização, juventudilização, pauperização e envelhecimento. Essas características evidenciam que não existem mais indivíduos particularmente vulneráveis ao vírus HIV, na medida em que pessoas em todas as fases do ciclo de vida estão expostas à contaminação. (LÔBO, 2011; SANTOS; ASSIS, 2011).

Considerando o contexto religioso, observou-se que $23,8 \%$ dos idosos julgavam a Aids como um castigo divino para aqueles que cometeram pecados. Lazzarotto et al. (2008), ao questionar idosos da região Sul do Brasil, constatou proporção semelhante (aproximadamente $21 \%$ ). A relação entre religião e ISTs é muito delicada, principalmente entre os idosos. De acordo com Seffner et al. (2008), ainda existem muitos questionamentos e embates no que concerne à relação entre as crenças religiosas, os códigos morais delas decorrentes e a inserção de práticas cotidianas que podem tornar o indivíduo mais vulnerável às ISTs, as quais necessitam ser estudadas.
O conhecimento em saúde no contexto gerontológico é um determinante para o risco comportamental e a percepção de vulnerabilidade, e, nesse sentido, esta pesquisa mostrou vários resultados importantes. Apesar das limitações da investigação, atribuídas principalmente à amostragem, que foi por conveniência, portanto intencional, conseguiu-se identificar as principais lacunas de conhecimento sobre HIV/Aids em indivíduos idosos usuários da rede pública de saúde nos domínios "conceito", "prevenção", "transmissão" e "vulnerabilidade".

Nessa perspectiva, nota-se uma grande carência de políticas direcionadas a essa população em particular, uma vez que, atualmente, o desenvolvimento de estratégias educativas - em sua maioria - continua sendo direcionado ao público jovem, às gestantes, aos usuários de droga, aos homossexuais e às profissionais do sexo. De fato, os idosos constituem um novo grupo que urge pela inclusão em programas e ações de promoção e prevenção das IST/HIV/Aids. (GODOY et al., 2008; POTTES et al., 2007; MONTOYA; WHITSETT, 2003).

Apesar desse cenário, a adoção de políticas públicas de saúde que concentrem sua atenção nesta população faz-se necessária para conter o avanço do HIV/ Aids entre os indivíduos com mais de 60 anos em nossa região. Tais políticas públicas de cunho preventivo devem levar em consideração aspectos psicológicos, socioeconômicos e culturais que interferem na vulnerabilidade desse grupo etário e devem dedicar-se à elucidação das principais dúvidas relacionadas ao HIV/à Aids para promover uma mudan- 
ça comportamental dos idosos, sobretudo, incentivando a prática de sexo seguro na terceira idade.

\section{Conclusão}

Considera-se que os objetivos estabelecidos para este estudo foram alcançados, visto que foi possível avaliar o conhecimento em relação ao HIV/à Aids por meio da abordagem dos conceitos, das formas de prevenção e de transmissão, bem como da vulnerabilidade em idosos da comunidade usuários dos serviços públicos de saúde.

Depois de realizada a pesquisa, pode-se afirmar que foram identificadas algumas lacunas de conhecimento sobre HIV/Aids na amostra de idosos avaliada, demonstrando a necessidade de estratégias educativas em saúde sobre a temática que concentrem suas atenções para esta faixa etária. Vale ressaltar que este estudo, após ter identificado o baixo nível de conhecimento sobre a temática em foco, possibilitou à equipe profissional de saúde elaborar futuras ações de prevenção a serem desenvolvidas no Programa de Atenção à Saúde do Idoso.

\section{Knowledge of the community-dwelling elderly on HIV/Aids: an epidemiological study in a primary health care system of Belém, Pará, Brazil}

\section{Abstract}

The world's population is aging slowly and in view of this has been noted on numerous behavioral changes in sexuality of older people and to this makes if neces- sary to better understand both the aging process as the social relationships of this group whereas the active sex life. With that, in the present study aimed to evaluate the level of knowledge of the elderly on HIV/Aids. It is a descriptive population-based study with quantitative-exploratory method which involved 310 elderly individuals of both sexes, attended in public health services of the city of Belém, Pará, Brazil. To the data collection was used a semi-structured questionnaire about HIV/ Aids, including general characteristics and information about concept, transmission, prevention, vulnerability and treatment of HIV/Aids. The results obtained in the research revealed the low level of knowledge in relation to concepts, transmission and vulnerability of HIV/Aids. In this way, the misconceptions demonstrate the necessity to implement specific educational programs to this age group in the public health network.

Keywords: HIV. Knowledge. Health of the elderly . Epidemiology

\section{Referências}

ABBAS, A. K.; LICHTMAN, A. H.; POBER, J. S. Cellular and molecular immunology. Philadelphia: W. B. Saunders Company; 2000.

BERTONCINI, B. Z.; MORAES, K. S.; KULKAMP, I. C. Comportamento sexual em adultos maiores de 50 anos infectados pelo HIV. DST - Jornal Brasileiro de Doenças Sexualmente Transmissíveis, Rio de Janeiro, v. 19, n. 2, p. 75-79, 2007.

BRASIL. Lei $\mathrm{n}^{\circ} 10.741$, de $1^{\circ}$ de outubro de 2003. Dispõe sobre o Estatuto do Idoso, e dá outras providências. Diário Oficial da União, Brasília, DF, 3 out. 2003.

- Ministério da Saúde. Programa Nacional de DST/AIDS. 2005. Disponível em: <http://www.aids.gov.br>. Acesso em: 21 dez. 2011. 
FERNANDES, F. C. L.; COUTINHO, E. S. F.; MATIDA, A. Conhecimentos e atitudes relativas à SIDA/AIDS em uma população de favela do Rio de Janeiro. Caderno de Saúde Pública, Rio de Janeiro, v. 8, n. 2, p. 176-182, 1992.

FERNANDES, J. C. L. Evolução dos conhecimentos, atitudes e práticas relativas ao HIV/ Aids em uma população de favela do Rio de Janeiro. Caderno de Saúde Pública, Rio de Janeiro, v. 14, n. 3, p. 575-581, 1998.

FERRO, S.; SALIT, I. HIV infections in patients over 55 years of age. Journal of Acquired Immune Deficiency Syndromes, New York, v. 5, n. 4, p. 348-353, 1992.

GODOY, V. et al. O perfil epidemiológico da Aids em idosos utilizando sistemas de informações em saúde do Datasus: realidades e desafios. DST - Jornal Brasileiro de Doenças Sexualmente Transmissíveis, Rio de Janeiro, v. 20, n. 1, p. 7-11, 2008.

IQBAL, M. Can we get Aids from mosquito bites? Journal of the Louisiana State Medical Society, Baton Rouge, v. 151, n. 8, p. 429-433, 1999.

LAZZAROTTO, A. R. et al. O conhecimento de HIV/Aids na terceira idade: estudo epidemiológico no Vale do Sinos, Rio Grande do Sul, Brasil. Ciência \& Saúde Coletiva, Rio de Janeiro, v. 13, n. 6, p. 1833-1840, 2008.

LEITE, M. T.; MOURA, C.; BERLEZ, E. M. Doenças sexualmente transmissíveis e HIV/ AIDS na opinião de idosos que participam de grupos de terceira idade, Revista Brasileira de Geriatria e Gerontologia, Rio de Janeiro, v. 10, n. 3, p. 339-354, 2007.

LÔBO, M. P. Vulnerabilidade ao HIV/Aids: representações sociais de idosos residentes em zona rural. 2011. 95 f. Dissertação (Mestrado em Saúde Pública) - Pós-Graduação em Enfermagem e Saúde, Universidade Estadual do Sudoeste da Bahia, Jequié, 2011.

MIYATA, D. F. et al. Políticas e programas na atenção à saúde do idoso: um panorama nacional. Arquivos de Ciências da Saúde da Unipar, Umuarama, v. 9, n. 2, p. 135-140. 2005.

MONTOYA, I.; WHITSETT, D. New frontiers and challenges in HIV research among older minority populations. JAIDS Journal of Acquired Immune Deficiency Syndromes, New York City, v. 33, n. 2, p. 218-221, 2003.

OLIVI, M.; SANTANA, R. G.; MATHIAS, T. A. Comportamento, conhecimento e percepção de risco sobre doenças sexualmente transmissíveis em um grupo de pessoas com 50 anos e mais de idade. Revista Latino-Americana de Enfermagem, Ribeirão Preto, v. 16, n. 4 , p. $76-84,2008$.

POTTES, F. A. et al. Aids e envelhecimento: características dos casos com idade igual ou maior que 50 anos em Pernambuco, de 1990 a 2000. Revista Brasileira de Epidemiologia, São Paulo, v. 10, n. 3, p. 338-351, 2007.

SALDANHA, A. A.; FELIX, S. M.; ARAÚJO, L. F. Representações sobre a Aids na velhice por coordenadoras de drupos da terceira idade. Psicologia-USF, v. 13, n. 1, p. 95-103, 2008.

SANTOS, N. J. et al. A Aids no estado de São Paulo: as mudanças no perfil da epidemia e perspectivas da vigilância epidemiológica. Revista Brasileira de Epidemiologia, São Paulo, v. 5, n. 3, p. 286-310, 2002.

SANTOS, A. F.; ASSIS, M. Vulnerabilidade das idosas ao HIV/AIDS: despertar das políticas públicas e profissionais de saúde no contexto da atenção integral: revisão de literatura. Revista Brasileira de Geriatria e Gerontologia, Rio de Janeiro v. 14, n. 10, p. 147-157, 2011.

SEFFNER, F. et al. Respostas religiosas à Aids no Brasil: impressões de pesquisa acerca da Pastoral de DST/Aids da Igreja Católica. Ciências Sociais e Religião, Porto Alegre, v. 10, n. 10, p. 159-180, 2008.

SOUSA, J. Sexualidade na terceira idade: uma discussão da Aids, envelhecimento e medicamentos para disfunção erétil. $D S T$ Jornal Brasileiro de Doenças Sexualmente Transmissíveis, Rio de Janeiro, v. 20, n. 1, p. 59-64, 2008.

VERAS, R. P.; CAMARGO, J. Idosos e universidade: parceria para qualidade de vida. In: VERAS, R. (Org.) Terceira idade: um envelhecimento digno para o cidadão do futuro. Rio de Janeiro: Relume-Dumará; UnATI/ UERJ, 1995. 